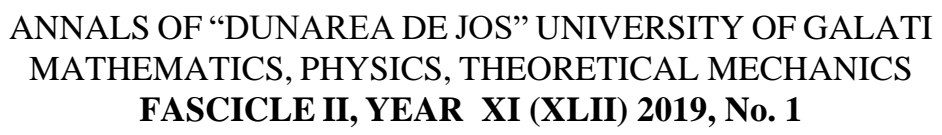

Article DOI : https://doi.org/10.35219/ann-ugal-math-phys-mec.2019.1.04

\title{
ANALYSIS OF COMMON MELANOMAS AND NEVI USING GEOMETRICAL FEATURES
}

\author{
Luminița Moraru ${ }^{1 *}$, Felicia Anișoara Damian², Simona Moldovanu ${ }^{3,4}$ \\ ${ }^{1 *}$ Faculty of Sciences and Environment, Physics Department, “Dunarea de Jos" University of Galati, \\ 47 Domneasca Street., RO-800008, Galati, Romania \\ ${ }^{2}$ Faculty of Science and Environment, "Dunarea de Jos" University of Galati, \\ 47 Domneasca Street, RO-800201, Galati, Romania \\ ${ }^{3}$ Faculty of Automation, Computers Sciences, Electronics and Electrical Engineering, "Dunarea de Jos" \\ University of Galati, 2 Stiintei Street, RO-800210, Galati, Romania \\ 4 ,'Dumitru Motoc" High School, 15 Milcov Street., RO-800509, Galati, Romania \\ *Corresponding author: luminita.moraru@ugal.ro
}

\begin{abstract}
This study aims to use some geometrical features to investigate and classify two common skin lesions, nevi and melanomas. Two sets of malignant and benign lesions were investigated; the first set contains 70 images from melanoma-diagnosed patients and the second set contains 100 of regular nevi images. A two-steps processing method is followed. First, segmentation of the skin lesions based on the optimal threshold determined by the histogram is performed, then a clustering operation using the Fuzzy C-Mean algorithm clusters the geometrical features. Asymmetry index, compactness, circularity and eccentricity were proposed as significant geometrical features. To validate the clustering results, the Euclidean distance between centroids was computed. Eccentricity well separates all objects under analysis and can differentiate between selected classes of skin lesions.
\end{abstract}

Keywords: geometrical features, clustering.

\section{INTRODUCTION}

Research over the last few years has confirmed that melanoma is the deadliest form of skin cancer that has a mortality rate of up to $65-70 \%$ among the population that is diagnosed with this type of skin cancer [1]. Melanoma is the $19^{\text {th }}$ most common type of skin cancer worldwide [2]. Both the Skin Cancer Foundation and the Melanoma Research Alliance claim that one person dies, in the world, every hour because of melanoma. The new American Cancer Society report [3] estimates that melanoma can be ranked as the fifth disease in the US, in point of the mortality rate, and it is estimated that 96,480 new cases of melanoma will be diagnosed in the near future. This is a 5.6\% rising in 2018 . These worrying data confirms that an early detection of melanoma increases the survival rate.

To analyze the skin lesions, a variety of image features have been proposed, among them the geometrical features [4], parameters related to the lesion contour [5, 6], features extracted from the histogram of the skin lesion image and features of the second order [7] are widely used. In 2013, Sadeghi et al. [8] have proposed a study based on lesion orientation, color and texture. Their study leads to a classification accuracy of 76.1\%. In 2013, Ramteke and Jain [9] implemented in MATLAB an algorithm that uses ABCD factors (asymmetry, border irregularity, color and diameter) and the TDS (total dermatoscopic score) for skin lesion classification. Using these features, the accuracy of malign skin lesion detection was improved to $90 \%$. Also, Jain et al. [10] showed that parameters such 
as asymmetry, contour irregularity, color and diameter can be used to classify normal skin and melanoma cancer lesion. Giotis et al. [11] proposed a decision support system to localize and extract the skin lesion and to compute the color and texture descriptors. A diagnostic accuracy of $81 \%$ is reported. In 2016, Jafari et al. [12], developed a set of color features to characterize different color characteristics of melanoma and achieved a classification rate of $76 \%$ for skin lesions using the variance, distribution and image color intensity. Chandrahasa et al. [13], used asymmetry, contour, color, diameter, and the second order characteristics (contrast, energy, correlation, homogeneity) features and demonstrated that color had the greatest ability to differentiate between regions in the image. Garg et al. [14] by using the ABCD rule and asymmetry, border irregularity, color and lesion diameter features have investigated the skin lesions.

In this paper, the same ABCD (Asymmetry, irregular Borders, Color, Diameter - lesions larger than $6 \mathrm{~mm}$ ) rule is used to investigate the skin lesions based on various geometrical features which can provide meaningful information about the irregularities of the skin lesions and their diameter. The proposed geometrical features will compensate the limited accuracy (between 59\% and $88 \%$ ) of $\mathrm{ABCD}$ rule in diagnosing melanoma. The following geometrical features, asymmetry (A), compactness (COMP), circularity (CIRC) and eccentricity (E) were computed. The image processing stages include skin lesion segmentation to separate the target lesion from skin [15], geometrical features extraction and classification by using Fuzzy C-Mean algorithm [16]. To analyze the clustering results [17], the Euclidean distance [18] as a validity measure between the centroids of the detected clusters is calculated.

\section{MATHEMATICAL APPROACHES}

a. Skin lesion segmentation

The segmentation stage is performed to separate the skin lesion from normal surrounding skin. The segmentation steps are described as: (i) the image is imported into the application; (ii) the color image is transformed into a gray level image; (iii) circumscribing an ellipse to the object; (iv) histogram of detected object is formed and the optimal threshold is established; (v) by using the optimum threshold, the elliptical mask is built; (vi) detect and extract the object from image and compute the features; (vii) the result of this algorithm is a vector of features AI, E, CIRC, COMP\}.

b. Asymmetry index (AI) belongs to shape features and allows to investigate the symmetry of the skin lesions. Benign lesions have a symmetrical shape and malignant lesions show an asymmetrical shape. AI is computed as [19]:

$$
\mathrm{AI}=\frac{\Delta \mathrm{A}}{\mathrm{A}} \times 100
$$

where, $\mathrm{A}$ is the total area of the image, $\Delta \mathrm{A}$ is the difference between the image area and the segmented lesion area.

c. Eccentricity (E) represents the elongation degree of an ellipse [20]:

$$
\mathrm{E}=\sqrt{1-\frac{R_{\min }}{R_{\max }}}
$$

where $R_{\min }$ and $R_{\max }$ are the minimum and maximum distances from the foci of the object to the contour of the object.

d. Circularity (CIRC) indicates how compact or elongated a region of interest is [21]:

$$
\mathrm{CIRC}=\frac{4 \pi \mathrm{A}}{\mathrm{P}^{2}}
$$


where, $\mathrm{P}$ denotes the perimeter of the lesion's border. Perimeter and area are measured by counting the pixel edge. An edge is viewed as a boundary between pixels inside the object and pixels outside the object. Area is the sum of pixels inside of the object.

e. Compactness (COMP) quantifies the degree to which an object (i.e. segmented skin lesion) is compact [22]:

$$
\mathrm{COMP}=\frac{\mathrm{P}^{2}}{4 \pi \mathrm{A}}
$$

f. The histogram of a monochrome image is defined by Ivanovich [23] as "the relative frequency of the gray level occurrence in the image." The same author mathematically defines the histogram as follows: equation:

For a $\mathrm{f}(\mathrm{x}, \mathrm{y})$ image, with a pixel size $\mathrm{M} \mathrm{x} \mathrm{N}$, the histogram is computed with the following

$$
\mathrm{h}(\mathrm{i})=\frac{1}{M N} \sum_{m=0}^{M-1} \sum_{n=0}^{N-1} \delta(i, f(m, n)), i=0, \ldots, L-1 ;,
$$

where $\delta$ is defined as:

$$
\delta(\mathrm{x}, \mathrm{y})=\left\{\begin{array}{lll}
1 & \text { dacă } & x=y \\
0 & \text { dacă } & x \neq y
\end{array}\right.
$$

Running the histogram pixel by pixel requires a gray level control, and the histogram function fulfills the condition [24]

$$
\sum_{i=0}^{L-1} h(i)=1
$$

g. The Fuzzy C-Means method (FCM) allows an element of the dataset to belong to two or more clusters. Assigning an object to a cluster is done through the degree of membership such as:

$$
u_{i j}=\frac{1}{\sum_{K=1}^{K}\left(\frac{\left(\left\|x_{i}-K_{j}\right\|\right)}{\left(\left\|x_{i}-K_{K}\right\|\right)}\right)^{\frac{2}{m-1}}}, K_{j}=\frac{\sum_{i=1}^{n} u_{i j}^{m} \mathrm{~g}_{i}}{\sum_{i=1}^{n} u_{i j}^{m}}
$$

FCM minimizes the following objective function:

$$
\mathrm{J}(\mathrm{U}, \mathrm{K})=\sum_{i=1}^{n} \sum_{j=1}^{k}\left(u_{i j}\right)^{m}\left\|x_{i}-K_{j}\right\|^{2}
$$

where, $\mathrm{x}=\left\{x_{1}, x_{2}, \ldots, x_{n}\right\}$ is the set of measured features; $\mathrm{K}=\left\{K_{1}, K_{2}, \ldots, K_{n}\right\}$ is the set of centroids and $k$ is the number of clusters; $\left\|x_{i}-K_{j}\right\|$ denotes a metric able to calculate the distance between an element and centroid of a cluster. $m \varepsilon[0, \infty)$ is the fuzzification degree and the value $\mathrm{m}=2$ was selected. $U=\left(u_{i j}\right)_{n g K}$ represents the matrix of the degree of membership; $u_{i j}$ is the degree of membership of an element $x_{i}$ to cluster $j$, which satisfies the following conditions:

$$
\begin{aligned}
& u_{i j} \varepsilon[0,1], \forall i=1, \ldots, n, \forall j=1, \ldots, k \\
& \sum_{j=1}^{k} u_{i j}=1, \forall i=1, \ldots, n
\end{aligned}
$$

h. Euclidean distance (ED) computes the distance between the centroids of the clusters [18]:

$$
E D=\sqrt{\left(x_{M}-x_{S}\right)^{2}+\left(y_{M}-y_{S}\right)^{2}}
$$

Where $\left(x_{M}, y_{M}\right)$ are the centroid's coordinates of the melanoma class and $\left(x_{S}, y_{S}\right)$ represents the centroid's coordinates of the nevi class. These coordinates were calculated for AI, E, CIRC and COMP features. 


\section{METHOD}

The framework of the proposed method consists of four blocks (see figure 1): (i) the image segmentation by using histogram threshold method; (ii) compute the features of the segmented objects: (iii) clustering data and ED calculation; and (iv) selection of the meaningful feature based on the maximal values of ED.
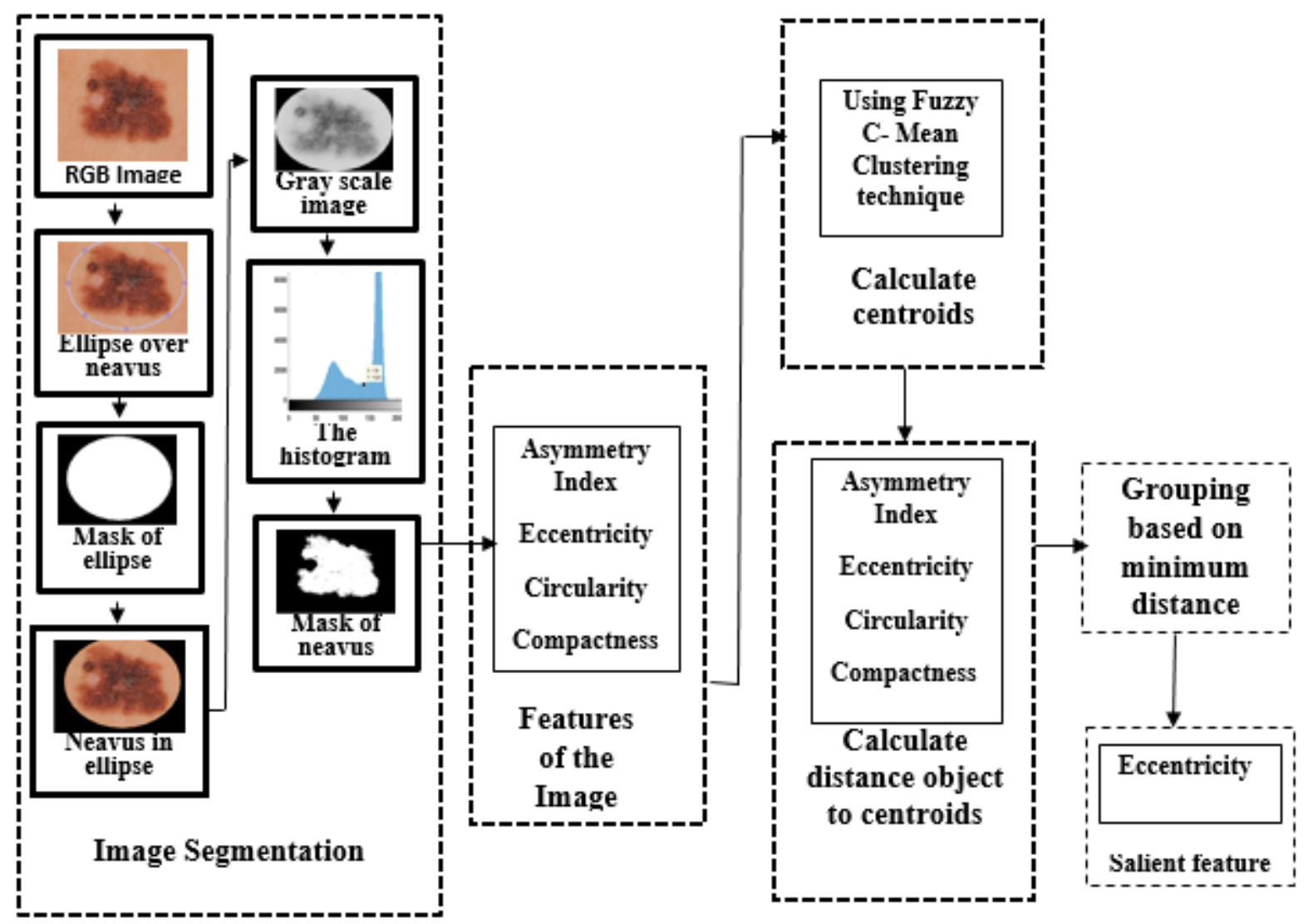

Figure 1. The framework of the proposed approach

The hardware and software platform was a computer with the following configuration: Processor: Intel (R) Core (TM) i3-6006U, CPU 1.99GHz, installed memory (RAM): 4Gb, system type: 64bit operating system-based processor and application software: MATLAB R2018a. The proposed method is tested on the images free downloaded from the Digital Archive of the Department of Dermatology of the University Medical Center in Groningen (UMCG), used for the development and testing of the MED-NODE system for the detection of skin cancer in macroscopic images. Two sets of malignant (70 images from melanoma-diagnosed patients) and benign lesions (100 images nevi images) were investigated.

\section{RESULTS AND DISCUSSIONS}

Figure 2 displays the results of the segmentation stage. The features of interest were computed and stored into a vector of features $\{$ AI, E, CIRC, COMP $\}$. Data associated to each feature over the all images in database are grouped using the FCM. The number of clusters was assumed to be $k=2$. Figure 3 shows the result of clustering and data in Table 1 illustrates the average ED values between the centroids. 

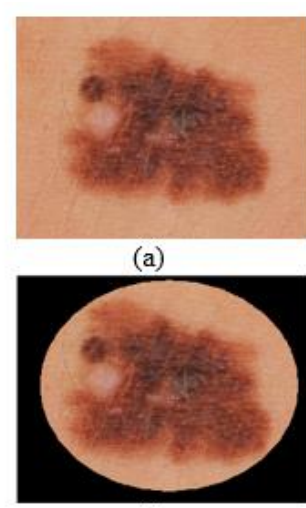

(d)

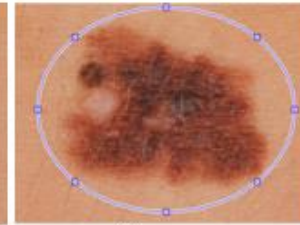

(b)

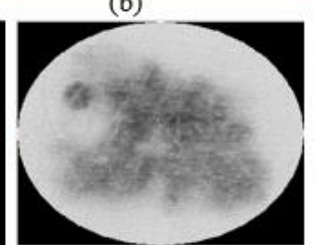

(e)

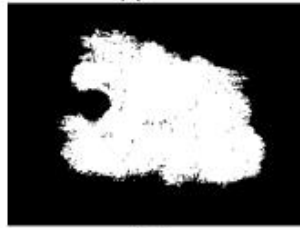

(g)

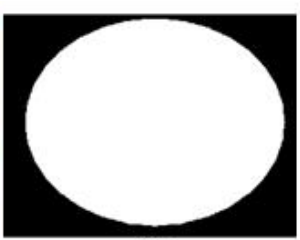

(c)

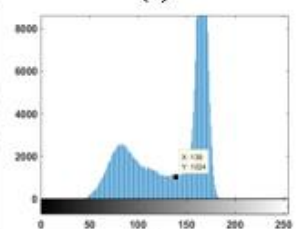

(f)

Figure 2. Segmentation results. (a) Original RGB image. (b) Ellipse circumscribed to the lesion. (c) The elliptical mask. (d) The elliptical mask containing the skin lesion. (e) The color image converted into a gray level image. (f) The histogram image. (g) Mask associated with skin lesion.

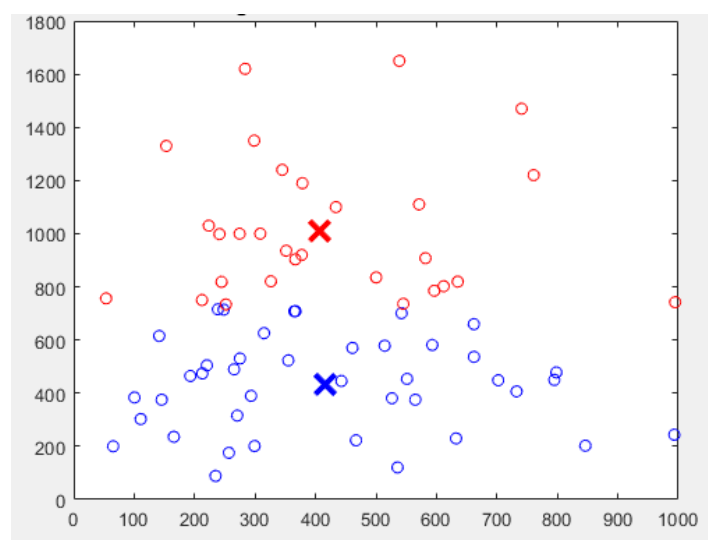

(a)

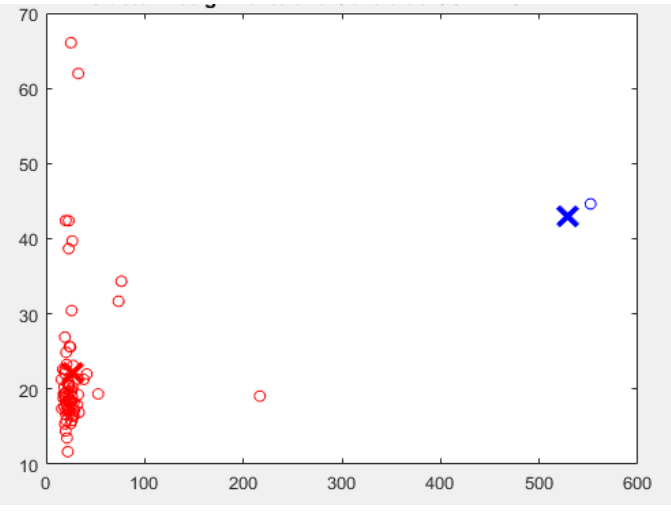

(c)

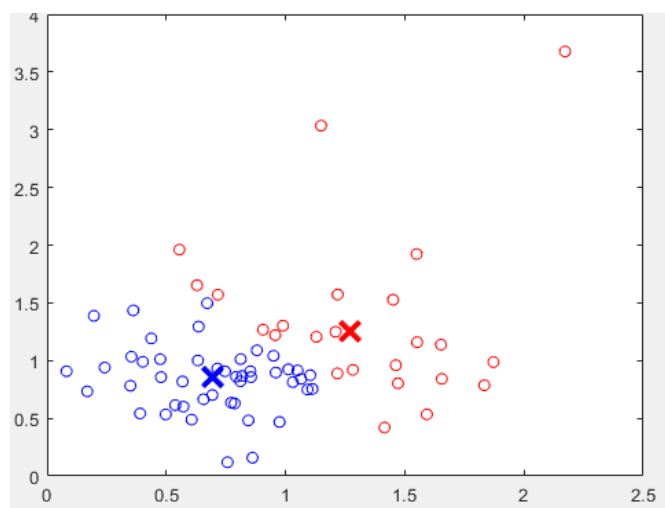

(b)

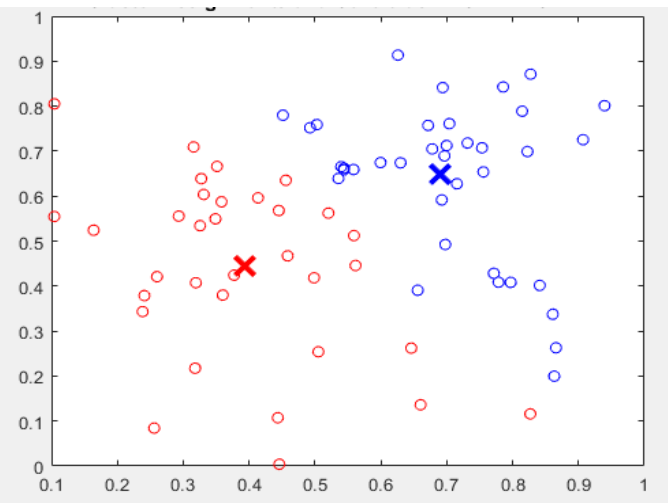

(d)

Figure 3. A comparison of the clustering for each geometrical feature. Red corresponds to melanoma, and blue to nevi. (a) CIRC; (b) AI; (c) COMP; (d) E. 
Table 1. The average ED values between the centroids of the detected clusters

\begin{tabular}{|l|l|l|l|}
\hline CIRC & AI & COMP & E \\
\hline $\mathbf{4 1 4 . 0 8}$ & $\mathbf{4 2 9 . 8}$ & 1155.02 & $\mathbf{5 7 7 . 2}$ \\
\hline
\end{tabular}

A better clustering solution is achieved when the distance between centroids is maximal. In this case, the more distinct the classes are then more relevant the features are. The results of the comparison shown in Fig. 3 indicate that for feature COMP (see Fig. 1(c)), no conclusions can be drawn on this experiment as the almost all objects are allocated to the melanoma class. In this case, the higher ED values are not a reliable indicator for clusters validity. The vector of features is reduced to \{AI, E, CIRC\}. All clusters are evenly distributed so, we cannot conclude which feature is more important for our declared goal. To rank the remaining features, the Euclidian distance is analyzed. From data in Table 1, we observed that a reasonably well separated clusters are for AI, CIRC and E features. This finding is interpreted as follows: two data belonging to the two different clusters will contribute to the classification task less if they were close separated than they are far away.

\section{CONCLUSIONS}

The problem analyzed in this paper is devoted to extracting subsets of geometric features which are most discriminative in skin lesions classification, simultaneously with clustering features. ED between centroids indicates the separability of clusters. The greater the distance is, the more relevant the features are. Experiments demonstrate that $\mathrm{E}$ feature provide a reasonably well separated clusters and is relevant in classification of healthy nevi from melanomas.

\section{References}

1. M. Petrescu, A. Alexandru, A. Benga, D. Dumitrescu, Difficulties of diagnosis and treatment in a case of malignant melanoma, Romanian Medical Journal - Vol. 62(3), pp.307-312, 2015.

2. F.Bray, J. Ferlay, I. Soerjomataram, R.L.Siegel, L.A.Torre, A.Jemal, Global Cancer Statistics 2018: GLOBOCAN estimates of incidence and mortality worldwide for 36 cancers in 185 countries, CA Cancer J Clin, in press.

3. M. Hurlbert, Estimated Rates of Melanoma Increasing in 2019, [online] Available: $<$ https://www.curemelanoma.org/blog/article/cancer-facts-and-figures-new-2019-numbersreleased-by-american-cancer-society>, [Accessed 17 March 2019].

4. S. Jain, V. Jagtap and N. Pise, "Computer aided melanoma skin cancer detection using image processing", Procedia Computer Science, pp. 736-741, 2015.

5. R. Garnavi, "Computer-aided diagnosis of melanoma," Ph.D. dissertation, University of Melbourne, Australia, 2011.

6. M. E. Celebi, H. Iyatomi, G. Schaefer, and W. V. Stoecker, "Lesion border detection in dermoscopy images," Computerised Medical Imaging and Graphics, vol. 33(2), pp. 148-153, 2009.

7. M. Lingala, R. Joe Stanley, R. K. Rader, J. Hagerty, H. S.Rabinovitz, M. Oliviero, and Stoecker, "Fuzzy logic color detection: Blue areas in melanoma dermoscopy images", Computerized Medical Imaging and Graphics, vol. 38(5), pp. 403-410, 2014.

8. M. Sadeghi, D.I. McLean, T.Lee, M.S. Atkins, Detection and analysis of irregular streaks in dermoscopic images of skin lesions, IEEE TRANSACTIONS ON MEDICAL IMAGING, Vol. 32(5), pp. 849-861, 2013.

9. N.S. Ramteke, S.V. Jain, ABCD rule based automatic computer-aided skin cancer detection using MATLAB®, Int. J. Computer Technology \& Applications, Vol. 4 (4), pp. 691-697, 2013.

10. S. Jain, V. Jagtap, N. Pise, Computer aided Melanoma skin cancer detection using Image Processing, International Conference on Intelligent Computing, Communication \& Convergence, Bhubaneswar, Odisha, India, pp.735-740, 2015. 
11. I. Giotis, S. Land, N. Molders, M. Biehl, M.F. Jonkman, N. Petkov, "MED-NODE: A Computer-Assisted Melanoma Diagnosis System using Non-Dermoscopic Images", Expert Systems with Applications. Vol. 42(19), pp.6578-6585, 2015.

12. M. H. Jafari, S. Samavi, S.M.R. Soroushmehr, H. Mohaghegh , N. Karimi , K. Najarian, Set of Descriptors for Skin Cancer Diagnosis using Non-Dermoscopic Color Images, pp.26382642, IEEE International Conference on Image Processing (ICIP), Phoenix, 2016.

13. M.Chandrahasa, V. Vadigeri, D. Salecha, Detection Of Skin Cancer Using Image Processing Techniques, International Journal of Modern Trends in Engineering and Research (IJMTER) Vol.3, pp.111-114, 2016.

14. N. Garg, P. Kaur, V. Sharma, Melanoma Skin Cancer Detection Using Image Processing, Chapter from book Sensors and Image Processing, (pp.111-119) Ed. Springer, Berlin, 2018.

15. L.Moraru, S. Moldovanu, A. Biswas, Optimization of breast lesion segmentation in texture feature space approach, Medical Engineering \& Physics Vol. 36, Issue 1, pp.129-135, 2014.

16. G. Gan, C. Ma, J. Wu, Data Clustering: Theory, Algorithms and Applications. Philadelphia: SIAM, Ed. ASA-SIAM, 2007.

17. D. Pascual, F. Pla, J.S. Sanchez.: Cluster validation using information stability measures, Pattern Recognition Letters 31, pp. 454-461, 2010.

18. E. Ceangă, I. Munteanu, A. Bratcu, M. Culea, Semnale, circuite și sisteme, Analiza semnalelor, Ed. Academica, Galați, 2001.

19. Chandrahasa M, Varun Vadigeri and Dixit Salecha, Detection of Skin Cancer using Image Processing Techniques, International Journal of Modern Trends in Engineering and Research, Vol. 03, Issue 05, pp.111-114, 2016.

20. Boccara, N., Essentials of Mathematica: With Applications to Mathematics and Physics, Springer-Verlag, New York, 2007.

21. Saket Kumar, Ajay Kumar Yadav, Detection of melanoma using asymmetric features, International Journal of Advanced Engineering Research and Technology (IJAERT), Vol. 4 Issue 11, pp.328-334, November 2016.

22. M. Messadi, A. Bessaid, A. Taleb-Ahmed, Extraction of specific parameters for skin tumour classification, Journal of Medical Engineering \& Technology, Vol. 33, No. 4, pp.288-295, May 2009.

23. M. Ivanovici, Procesarea Imaginilor - Îndrumar de laborator, Editura Universităţii Transilvania, Brașov, 2006.

24. S. Moldovanu, L. Moraru, M. C. Nicolae, Comparative Analysis of Ultrasound Image using Gray Level, Annals of Dunarea de Jos University, Fascicle II, Anul II,Vol. 2, 145-151, 2010. 